This is the author's final, peer-reviewed manuscript as accepted for publication. The publisher-formatted version may be available through the publisher's web site or your institution's library.

\title{
Light scattering shape diagnostics for nano-agglomerates
}

George W. Mulholland, Lei Zhou, Michael R. Zachariah, William R. Heinson, Amit Chakrabarti, Christopher Sorensen

\section{How to cite this manuscript}

If you make reference to this version of the manuscript, use the following information:

Mulholland, G. W., Zhou, L., Zachariah, M. R., Heinson, W. R., Chakrabarti, A., \& Sorensen, C. (2013). Light scattering shape diagnostics for nano-agglomerates. Retrieved from http://krex.ksu.edu

\section{Published Version Information}

Citation: Mulholland, G. W., Zhou, L., Zachariah, M. R., Heinson, W. R., Chakrabarti, A., \& Sorensen, C. (2013). Light scattering shape diagnostics for nano-agglomerates. Aerosol Science and Technology, 47(5), 520-529.

Copyright: Copyright (C) American Association for Aerosol Research

Digital Object Identifier (DOI): doi:10.1080/02786826.2013.767435

Publisher's Link:

http://www.tandfonline.com/doi/abs/10.1080/02786826.2013.767435\#.UYQv4Er7BoE

This item was retrieved from the K-State Research Exchange (K-REx), the institutional repository of Kansas State University. K-REx is available at http://krex.ksu.edu 


\title{
Light Scattering Shape Diagnostics for Nano-Agglomerates
}

George W. Mulholland, ${ }^{1,2}$ Lei Zhou, ${ }^{2}$ Michael R. Zachariah ${ }^{1,2}$, William R. Heinson ${ }^{3}$, Amit Chakrabarti ${ }^{3}$, Christopher Sorensen ${ }^{3}$

${ }^{1}$ University of Maryland, College Park, MD

${ }^{2}$ National Institute of Standards and Technology, Gaithersburg, MD

${ }^{3}$ Kansas State University, Manhattan, KS

Address correspondence to: George W. Mulholland, Department of Mechanical Engineering, University of Maryland, 2181 Glenn L. Martin Hall, College Park, MD 20742, USA. Email: georgewm@umd.edu

\begin{abstract}
Motivated by light scattering experiments showing enhanced intensity of electric field aligned nano-agglomerates vs. randomly oriented nano-agglomerates, we address the theoretical basis for this effect by applying the theory of small angle Rayleigh-DebyeGans light scattering to oriented nano-clusters generated by classical diffusion-limited cluster-cluster aggregation (DLCA). Based on more than 100 nano-clusters with 30 monomers and with 100 monomers, the ratio of the slopes of the inverse of the structure factor vs. the momentum transfer squared $\left(S(q)^{-1}\right.$ vs. $\left.q^{2}\right)$ for the partially aligned (aligned along the major axis but free to rotate about that axis) and randomly oriented clusters is well correlated with a linear fit to the shape anisotropy, defined as the ratio of the square of the major to minor principle radii of gyration. It is also shown that state of the art small-angle aerosol scattering measurements would have the angular resolution required to measure the shape anisotropy with 30 to 1000 nano-monomers with a size parameter of 0.15 .
\end{abstract}

For large q for nano-clusters with 30 to 1000 monomers, it is shown from the simulations that $S(q)$ for the partially aligned clusters is not proportional to $q^{-D_{f}}$, where $D_{f}$ is the fractal dimension, as it is for randomly oriented clusters. Nano-clusters with a fixed orientation are shown to result in a structure factor with multiple peaks, which could be used to obtain more detailed information about particle structure than shape anisotropy.

The measurements reported in the literature showing enhanced scattering for partially aligned soot agglomerates were for angle integrated measurements. Calculation of the integrated light scattering cross section for the same range of angles and polarization direction as the experiments indicate a significant enhancement of $70 \%$ and $120 \%$ for two representative aspect ratios. The smaller value overlaps with measured values of the scattering enhancement for oriented soot agglomerates in an electric field.

\section{INTRODUCTION}

Theoretical predictions of the light scattering by fractal aerosol nano-agglomerates have focused on the orientationally averaged scattering behavior (Martin and Hurd 1987, Mountain and Mulholland 1988, Sorensen 2001). This is appropriate for most aerosol 
experiments because the rotation time of the agglomerate is fast compared to the time for the scattering measurement. Even for cases where the measurement is fast, there are typically a large number of agglomerates in the scattering volume each with a random orientation. So again the random orientation is a good approximation. The prefix nano refers to the monomer particle diameter $D$ being less than about $40 \mathrm{~nm}$ or size parameter $(\pi D / \lambda)$ less than 0.2 , where $\lambda$ is the wavelength of light.

Such orientationally averaged measurements do not allow the determination of the shape of the agglomerates. There have been measurements of the light scattering by nanoagglomerates, both randomly oriented and partially aligned in an electric field. These measurements have been made for smoke from a variety of fuels, smoke from Kuwait oil fires, and iron oxide chain-aggregates by Colbeck et al. (1997), Chen et al. (1991), and Weiss et al. (1992), respectively. For both of the smoke studies, the authors found that for the fractal agglomerate smoke (soot), the total scattering coefficient increased by 20 $\%$ to $70 \%$ for the aligned soot relative to the randomly oriented soot. For the iron oxide chain aggregates, explicit results for the change in the scattering intensity are not given. However, data from Fig. 5 (Chen et al. 1991) for $2.9 \mu \mathrm{m}$ long chains with $0.06 \mu \mathrm{m}$ primary sphere diameters indicates a seven fold $(700 \%)$ increase in the light scattering intensity.

The multi-angle light scattering spectrometer developed by Dick (2006) is able to simultaneously measure the light scattering intensity at 12 polar angles in a time interval of about $3 \mu \mathrm{s}$. This time is small compared to the rotation time of $1 \mathrm{~ms}-5 \mathrm{~ms}$ for the smoke agglomerates studied by Colbeck et al. and Weiss et al. This suggests that it may be feasible to obtain light scattering data for a single agglomerate in a single orientation. This instrument could potentially provide more information on the particle structure, since there is no orientation averaging or averaging over a population of particles.

The theory of small angle light scattering (Guinier 1939, Zimm 1948, Tanford 1961, Kerker 1969) relates the orientation averaged light scattering from a particle as a function of scattering angle to the radius of gyration of the particle. Here we extend this analysis to the case of light scattering by a nano-cluster aligned along one of it principal axes. We show how the small angle light scattering structure factor, $S(q)$, of the aligned and randomly oriented agglomerate can provide information on the shape of the particle. The key quantity is the slope ratio $S R$, which is equal to the ratio of the slopes of $S^{-1}(\mathrm{q}) \mathrm{vs} . q^{2}$ for the oriented agglomerate versus the randomly oriented agglomerate. We show how $S R$ is related to the anisotropy of the agglomerate. The small angle analysis is also applied to the case of an agglomerate in one fixed orientation.

To assess the feasibility of this approach, diffusion limited cluster-cluster aggregation simulations (DLCA) were carried out producing clusters with sizes up to 1000 monomers. The value of $S R$ was computed for these simulated clusters and the relation between $S R$ and the shape anisotropy was determined.

The large $q$ behavior of the structure factor was computed for randomly oriented nanoclusters, for nano-clusters rotationally averaged about one axis, and for nano-clusters in a 
fixed orientation. Integrated scattering cross sections are computed for simulated clusters and compared with the measurements of Colbeck et al. (1997) and Weiss et al. (1992).

\section{THEORY}

\section{Small Angle Light Scattering for Oriented Agglomerates}

The differential scattering cross section for a cluster with $\mathrm{N}$ nano-monomers, $d \sigma^{a g g}(\mathbf{q}) / d \boldsymbol{\Omega}$, can be expressed in terms of the Rayleigh scattering cross section of the monomer and the structure factor, $S(\mathbf{q})$, of the monomer (Sorensen 2001 and Mountain and Mulholland 1988):

$$
\frac{d \sigma^{a g g}(\mathbf{q})}{d \Omega}=N^{2} \frac{d \sigma^{m}}{d \Omega} S(\mathbf{q})
$$

The Rayleigh scattering for the monomer for the polarization direction of the incident light perpendicular to the scattering plane is given by:

$$
\frac{d \sigma^{m}}{d \Omega}=k^{4} a^{6}\left|\frac{m^{2}-1}{m^{2}+2}\right|^{2}
$$

The quantity $m$ is the complex refractive index, $a$ the monomer radius, and $k$ equals $2 \pi / \lambda$. The structure factor is given by a sum over the $N$ monomers in the agglomerate (Sorensen 2001):

$S(\mathbf{q})=\frac{1}{N^{2}}\left|\sum_{j=1}^{N} e^{-i \boldsymbol{q} \cdot \mathbf{r}_{j}}\right|^{2}=\frac{1}{N^{2}}\left[\sum_{j=1}^{N} e^{-i q \cdot r_{j}}\right]\left[\sum_{l=1}^{N} e^{i \boldsymbol{q} \cdot r_{l}}\right]$,

The momentum transfer vector $\mathbf{q}$ is expressed in terms of the scattered and incident momentum transfer vectors, $\mathbf{k}_{s}$ and $\mathbf{k}_{\boldsymbol{i}}$.

$$
\begin{aligned}
& \mathbf{q}=\mathbf{k}_{\mathrm{s}}-\mathbf{k}_{\mathbf{i}} \\
& |\mathbf{k}|=k=2 \pi / \lambda \\
& |\mathbf{q}|=\frac{4 \pi}{\lambda} \sin (\theta / 2)
\end{aligned}
$$

Expanding the exponentials in Eq.(3) for small $\mathbf{q} \cdot \mathbf{r}_{\mathrm{j}}$, one obtains the following expression for S(q) to order $\left(\mathbf{q} \cdot \mathbf{r}_{\mathrm{j}}\right)^{2}$ :

$$
S(\mathbf{q})=1-\frac{1}{N} \sum_{j=1}^{N}\left(\mathbf{q} \cdot \mathbf{r}_{\mathbf{j}}\right)^{2}+\frac{1}{2 N^{2}} \sum_{j=1}^{N} \mathbf{q} \cdot \mathbf{r}_{\mathbf{j}} \sum_{l=1}^{N} \mathbf{q} \cdot \mathbf{r}_{\mathbf{l}}
$$

The first sum in the last term in Eq.(7) can be expressed as:

$$
\sum_{j=1}^{N} \mathbf{q} \cdot \mathbf{r}_{\mathbf{j}}=\mathbf{q} \cdot \sum_{j=1}^{N} \mathbf{r}_{\mathbf{j}}=N \mathbf{q} \cdot \mathbf{r}_{\mathbf{c m}}
$$


Our coordinate system is defined relative to the center of mass; thus the last term in Eq.(7) vanishes so that:

$$
S(\mathbf{q})=1-\frac{1}{N} \sum_{j=1}^{N}\left(\mathbf{q} \cdot \mathbf{r}_{\mathbf{j}}\right)^{2}
$$

We are interested in the behavior of $\mathrm{S}(\mathrm{q})$ for clusters with either a fixed orientation with their long axis perpendicular to the scattering plane, or the same situation but with rotational averaging about the long axis and finally for a randomly oriented clusters. The incident light propagates in the $\mathrm{z}$ - direction, the polarization and alignment directions are along the $\mathrm{x}$-axis, and the scattering plane is the $\mathrm{y}-\mathrm{z}$ plane as shown in Figure 1. For small scattering angles, $\mathbf{q}$ is given by:

$$
\mathbf{q}=\frac{4 \pi}{\lambda} \sin (\theta / 2)\left[-\widehat{\mathbf{u}}_{\mathbf{z}} \sin (\theta / 2)+\widehat{\mathbf{u}}_{\mathbf{y}} \cos (\theta / 2)\right] \cong \frac{4 \pi}{\lambda} \sin (\theta / 2) \widehat{\mathbf{u}}_{\mathbf{y}}=q \widehat{\mathbf{u}}_{\mathbf{y}}
$$

where $\mathbf{u}$ represents a unit vector. The component of $\mathbf{q}$ in the $x$ direction is negligible for small $\theta$. Substituting Eq.(10) in Eq.(9), we obtain:

$$
S(q)=1-\frac{q^{2}}{N} \sum_{j=1}^{N} y_{j}^{2}=1-q^{2} Y^{2}
$$

The quantity $Y$ is the y-component of the radius of gyration of the cluster. We shall use the notation $S_{f}$ to denote the structure factor for a cluster in a fixed orientation.

For the partially aligned case, the x-axis is assumed to be along the major principal axis of the agglomerate. The cluster is aligned but free to rotate about the $\mathrm{x}$-axis. The expression for the aligned structure factor is given by:

$$
\langle S(\mathbf{q})\rangle_{\alpha}=1-\frac{1}{N} \sum_{j=1}^{N}\left\langle\left(\mathbf{q} \cdot \mathbf{r}_{\mathbf{j}}\right)^{2}\right\rangle_{\alpha}
$$

The change in coordinates of the monomers resulting from a rotation of angle $\alpha$ about the $\mathrm{x}$-axis is given by:

$$
\left(\begin{array}{c}
x_{j}^{r} \\
y_{j}^{r} \\
z_{j}^{r}
\end{array}\right)=\left(\begin{array}{ccc}
1 & 0 & 0 \\
0 & \cos \alpha & \sin \alpha \\
0 & -\sin \alpha & \cos \alpha
\end{array}\right)\left(\begin{array}{c}
x_{j} \\
y_{j} \\
z_{j}
\end{array}\right)
$$

Using Eqs. (11) and (13) we obtain the following expression:

$\mathbf{q} \cdot \mathbf{r}_{\mathbf{j}}^{\mathbf{r}}=q\left[\cos \alpha y_{j}+\sin \alpha z_{j}\right]$

Substituting Eq.(14) into Eq.(12), we obtain:

$$
\langle S(q)\rangle_{\alpha}=1-\frac{1}{N} q^{2} \sum_{j=1}^{N} \frac{\int_{0}^{2 \pi}\left[\cos ^{2} \alpha y_{j}^{2}+\sin ^{2} \alpha z_{j}^{2}+2 \sin \alpha \cos \alpha y_{j} z_{j}\right] \mathrm{d} \alpha}{\int_{0}^{2 \pi} \mathrm{d} \alpha}
$$


Carrying out the integrations and performing the sum leads to:

$$
\langle S(q)\rangle_{\alpha}=1-\frac{1}{2} q^{2}\left(Y^{2}+Z^{2}\right)
$$

We shall use the shorthand notation $S_{\mathrm{a}}(q)$ for the structure factor of the partially aligned cluster (rotation about the $x$ - axis). The quantity $\boldsymbol{Z}$ is the $z$ component of the radius of gyration.

The third case is for a random orientation where the agglomerate is rotated through the solid angle $4 \pi$. It is convenient to use a coordinate system with $\mathbf{q}$ in the $\mathrm{z}$ direction with $\mathbf{r}_{\mathrm{j}}$ oriented with polar angle $\theta_{l}$ and azimuthal angle $\varphi_{l}$, where the subscripts are used to differentiate from the scattering angle $\theta$. The orientation of the q-r dot product is computed as follows:

$\left\langle\sum_{j=1}^{N}\left(\mathbf{q} \cdot \mathbf{r}_{\mathbf{j}}\right)^{2}\right\rangle=\sum_{j=1}^{N} q^{2} r_{j}^{2}\left\langle\cos ^{2} \theta_{1 j}\right\rangle$

The orientation average is computed as the integration over $\theta_{1}$ and $\varphi_{1}$.

$$
\left\langle\cos ^{2} \theta_{1 j}\right\rangle=\frac{\int_{0}^{2 \pi} \int_{0}^{\pi} \cos ^{2} \theta_{1 j} \sin \theta_{1 j} \mathrm{~d} \theta_{1 j} \mathrm{~d} \phi_{1 j}}{\int_{0}^{2 \pi} \int_{0}^{\pi} \sin \theta_{1 j} \mathrm{~d} \theta_{1 j} \mathrm{~d} \phi_{1 j}}=\frac{1}{3}
$$

From Eqs.(9), (17), and (18), we find that

$$
\langle S(\mathbf{q})\rangle_{\theta_{1}, \phi_{1}}=1-\frac{q^{2}}{3 N} \sum_{j=1}^{N} r_{j}^{2}=1-\frac{1}{3} q^{2} R_{g}^{2}
$$

In this case we will use the shorthand notation $S_{\mathrm{r}}(q)$ for the random orientation average.

The key results of this section are Eqs.(11), (16), and (19). A simple test for the validity of these equations is to consider a spherical particle. In this case, all three equations should give the same result because $X^{2}=Y^{2}=Z^{2}$. This is true since $S_{f}(q), S_{a}(q)$, and $S_{r}(q)$ are all equal to $1-q^{2} X^{2}$.

\section{Relationship between the Small Angle Structure Factor and Agglomerate Shape Anisotropy}

Agglomerate shape is described by the inertia tensor (Fry et al. 2004). For a threedimensional ( $3 d$ ) body of $N$ discrete and equal masses the inertia tensor is

$$
T=\sum_{j=1}^{N}\left(\begin{array}{ccc}
y_{j}^{2}+z_{j}^{2} & -x_{j} y_{j} & -x_{j} z_{j} \\
-x_{j} \mathrm{y}_{\mathrm{j}} & x_{j}^{2}+\mathrm{z}_{j}^{2} & -y_{j} \mathrm{z}_{j} \\
-x_{j} z_{j} & -y_{j} z_{j} & x_{j}^{2}+y_{j}^{2}
\end{array}\right)
$$


Diagonalizing $T$ and dividing by the number of monomers $N$, one obtains the square of principle radii of gyration $R_{j}^{2}$ for $j=1,2,3$ with $R_{1} \leq R_{2} \leq R_{3}$. Anisotropy is used as a measure of cluster "stringiness" and can be defined by the ratio of the squares of principle radii of gyration (Fry et al. 2004):

$A_{i j}=\frac{R_{i}^{2}}{R_{j}^{2}}$

where $i, j=1,2,3$. Here we focus on the ratio $A_{31}$, which is the ratio of the largest to the smallest principle radii of gyration. The radius of gyration $R_{g}$ is used as a measure of the overall cluster size which is related to the principle radii of gyration $R_{i}^{2}$ in the following way:

$R_{g}^{2}=\frac{1}{2}\left(R_{1}^{2}+R_{2}^{2}+R_{3}^{2}\right)$.

Here we define another measure of shape anisotropy the slope ratio, $S R$, which is the ratio of slopes of $S(q)^{-1}$ vs. $q^{2}$ for the random orientation (Eq.(19)) to the partially aligned orientation (Eq.(16)).

$S R=\frac{2}{3} \frac{\left(X^{2}+Y^{2}+Z^{2}\right)}{\left(Z^{2}+Y^{2}\right)}$

From Eq.(11) and the definition of the principle radii of gyration, one finds the following relationships between $R_{1}, R_{2}$, and $R_{3}$ and $X, Y$, and $Z$.

$$
\begin{aligned}
& R_{1}^{2}=Y^{2}+Z^{2} \\
& R_{2}^{2}=Z^{2}+X^{2} \\
& R_{3}^{2}=Y^{2}+X^{2}
\end{aligned}
$$

From these 3 equations together with the definitions of $S R$ and $A_{31}$, one obtains the following relationship:

$$
S R=\frac{2}{3}\left(A_{31}+\frac{X^{2}}{X^{2}+Y^{2}}\right)
$$

The quantity $A_{31}$ is always greater than 1 while the $2^{\text {nd }}$ term is always smaller than 0.5 because $X$ is the smallest component of the radius of gyration. In a later section we will analyze the correlation between $S R$ and $A_{31}$ for clusters ranging in size from 10 to 1000 monomers.

\section{SIMULATION}

Clusters used in this work were obtained from an off-lattice diffusion limited cluster aggregation (DLCA) algorithm (Fry et al. 2004) using an efficient link-list method (Allen and Tildesly 1987). DLCA simulations successfully model aggregates formed in aerosols and colloids which yield $D_{f}=1.8$ (Family and Landau 1984; Jullien and Botet 1987; 
Meakin 1999). These simulations started with $10^{6}$ particles at a monomer volume fraction of $f_{v}=0.001$ that were randomly placed in a three dimensional box. At the beginning of each time step, the number of clusters $\left(N_{c}\right)$ was counted (note that the number of monomers was included in $N_{c}$ ). A random cluster was chosen and time was incremented by $N_{c}^{-1}$. The probability that the cluster moved was inversely proportional to that cluster's radius of gyration $\left(p \propto R_{g}^{-1}\right)$ and was normalized so that monomers had $p=1$. Clusters moved in random directions a distance of one monomer diameter. When two clusters collided, they irreversibly stuck together, and $N_{c}$ was decremented by 1 . The resulting cluster sizes ranged from a few monomers to more than 1000. Rotational motions of the clusters were neglected in these simulations.

\section{CALCULATIONS}

The output of the simulations includes the coordinates of each monomer in the cluster. The inertia tensor, Eq.(20), was diagonalized for each cluster giving the principal radii (eigenvalues) and their orientation (eigenvectors) relative to the space fixed coordinate system. For each cluster the radius of gyration $R_{g}$, the anisotropy $A_{31}$, and the slope ratio SR are computed from the principal radii of gyration using Eqs. (21) to (26). In these calculations, the radius of the monomer is assumed to be $20 \mathrm{~nm}$.

The structure factor is computed for a scattering geometry illustrated in Figure 1. The incident light with an assumed wavelength of $405 \mathrm{~nm}$ is propagating in the $x$-direction and is linearly polarized in the $z$-direction. The assumed monomer size and wavelength are typical of the conditions for light scattering measurements of soot though larger monomer sizes and wavelengths are also common. The scattering plane is the $y-z$ plane with scattering angle $\theta$. The clusters are oriented with the major axis, $R_{3}$, in the $x$ direction and the minor axes $R_{1}$ is aligned in the $z$-direction. The orientation is shown for an idealized cluster in Figure 1. Based on the monomer coordinate location relative to the principal axis coordinate system with origin at the center of mass, the structure factor is computed using Eq.(3) and (6) as a function of $q$ for angles ranging from $0^{\circ}$ to $180^{\circ}$. For the partially aligned orientation, the value of $S(q)$ is computed with rotations every $10^{\circ}$ as the cluster is rotated about the z-axis. The average of these values is denoted as $S_{a}(q)$. The random orientation average is obtained by computing $S(q)$ for 216 orientations covering the full range of Euler angles for the coordinate axes for a fixed scattering angle. The average of these values is denoted as $S_{r}(q)$.

The results shown in the following section are expressed as $1 / S(q)$, which is the Zimm plot formalism and has been widely used for fractal aerosol agglomerates. For small $\mathrm{q}$ the asymptotic expression based on Eqs.(16) and (19) are:

$$
\begin{aligned}
& 1 / S_{a}(q)=1+\frac{1}{2} q^{2}\left(Z^{2}+Y^{2}\right) \\
& 1 / S_{r}(q)=1+\frac{1}{3} q^{2} R_{g}^{2}
\end{aligned}
$$


A key issue in analyzing the results is the range of $q$ over which the computed $S(q)$ is described by the above equation. This is important in assessing the feasibility of quantitatively determining $S R$ defined by Eq.(23) by light scattering experiments.

\section{RESULTS}

\section{Small q Behavior of Structure Factor}

The structure factors were computed for clusters with nominally 30 spheres, 100 spheres, 300 spheres, and 1000 spheres generated by the DLCA described above. Five clusters were selected for each of these cluster sizes, with $A_{31}$ ranging from about 2 to 6 . The $x-z$ projected image of 300 sphere clusters, with $A_{31}$ equal to 2 and 6 are shown in Figure 2 to illustrate the range of aspect ratios considered in this study. For the 300 sphere clusters, the average value of $A_{31}$ is about 4.0 and the standard deviation is about 2.0. This range contains a majority of the clusters though the population is broad with 3 of the 137 clusters with values of $A_{31}$ larger than 10 .

The effect of alignment on $1 / S(q)$ is shown for the 300 sphere clusters in Figure 3 . It is seen that the Zimm-type plots give a linear dependence of $1 / S(q)$ on $q^{2}$ as predicted by Eqs.(28) and (29). This plot shows that the partially aligned scattering is correlated with the anisotropy $A_{31}$ with the slope decreasing by about a factor of 3 for particle 3 relative to particle 1 as $A_{31}$ increases by about a factor of 3 . It is also apparent from Figure 3 that there is a much smaller variation in the slope for the random orientation as $A_{31}$ is changed.

A closer inspection indicates that there is a slight upward curvature in the plots. This is evident from the deviation between the calculated points and the visual linear fit to the data for particle 3 for $1 / S(q)<1.2$. The slope ratio $S R$, defined by Eq.(23), is equal to the ratio of the slope for the randomly oriented cluster to the aligned cluster in the limit as $q$ approaches zero. Of course, such a limit can not be reached experimentally. We have simulated possible experimental observation by making visual linear fits to the random and aligned orientations and then computing the ratio. We find that $S R$ computed from the visual fits agrees within $3 \%$ with the exact value of $S R$ computed using the coordinates of the cluster (Eq.(23)) for the three clusters shown in Figure 3. Table 1 compares the visual fit with the exact results for 10 different clusters, ranging in size from 30 monomer to 1000 monomers, and the largest discrepancy is $6 \%$. This demonstrates the potential for obtaining shape information from precise small angle scattering measurements for aligned and randomly oriented clusters. As the cluster size increases, the range of $q$ for linear $1 / S(q)$ vs. $q^{2}$ decreases. For random orientation, the range of $q$ decreases about six-fold from $1.2 \times 10^{-2} \mathrm{~nm}^{-1}$ to $2 \times 10^{-3} \mathrm{~nm}^{-1}$ as the cluster size increases from 30 monomers to 1000 monomers. The angle range for all the computed values of $S(q)$ corresponding to a $20 \%$ increase in $1 / S(q)$ is $7^{\circ}$ to $83^{\circ}$. Estimating $S R$ from experimental data would require at least three points distributed uniformly with regard to $q^{2}$. This would require forward scattering measurements at angles as small as about $2^{\circ}$. 
The correlation between $S R$ and $A_{31}$ was studied by plotting $S R$ vs. $A_{31}$ for a total of 227 clusters with 30 monomers and for 137 clusters with 300 monomers generated by DLCA. A good correlation is evident from Figure 4. Linear regression leads to slopes that agree within $1 \%$ :

$S R=0.166+0.671 A_{31}, \mathrm{R}=0.9992$ for 30 monomer clusters

$S R=0.161+0.675 A_{31}, \mathrm{R}=0.9988$ for 300 monomer clusters

The deltas between the value of $S R$ from the cluster coordinates and the regression line given by Eq.(31) are randomly distributed about zero and the standard deviation of the deltas is 0.062 . These regression lines underestimate the limiting value of $S R$ equal 1 for $A_{31}$ equal 1. It was expected that a bias would be evident for small values of $A_{31}$; however, we see only a hint of a bias in the deviation plot for the smallest values.

The regression expressions are consistent with Eq.(27). The slopes are within $1 \%$ of value of the slope in Eq.(27). The second term in Eq.(27) can vary at most from a value of $0(X=0)$ and $0.333(X=Y)$. The average of these two values is within $4 \%$ of the two regression intercepts.

\section{Large $q$ Behavior of Structure Factor}

The large $q$ behavior of the structure factor is widely used for determining the fractal dimension of nano-agglomerates both as aerosols and as colloidal suspensions. The results of many of these studies are summarized by Sorensen (2001). The large q behavior is shown in Figure 5 for 4 clusters with sizes ranging from 30 monomers to 1000 monomers. The clusters were selected so that the anisotropy, $A_{31}$, for each cluster was close to 4.0. For large $q$, the structure factors for the randomly oriented 300 and 1000 monomer cluster have a linear dependence on $q$ for the $\log$-log plot with a slope of about -1.8. This is a characteristic value seen in simulations and experimentally for diffusion limited cluster-cluster aggregation as discussed by Sorensen (2001). It is noteworthy that the aligned nano-agglomerates do not show this behavior. For the 1000 sphere cluster there is a reasonably constant slope of about 3.2 for q between $0.01 \mathrm{~nm}^{-2}$ and $0.02 \mathrm{~nm}^{-2}$, but then $S(q)$ becomes nearly constant as q approaches 0.3 . For the 300 monomer cluster $\mathrm{S}(q)$ has a bowed appearance with a slope of about 3.0 for $q$ in the range $0.02 \mathrm{~nm}^{-2}$ to $0.03 \mathrm{~nm}^{-2}$.

\section{DISCUSSION}

Currently there are no experimental data to compare our results for small angle measurements of aligned vs. random nano-agglomerates. However, Colbeck et al. (1997) and Weiss et al. (1992) have measured integrated light scattering from soot agglomerates in a random orientation and then in a partially aligned position with an electric field applied. The light scattering measurement was made with a reciprocal nephelometer (Fig. 6), which collects scattered light from $7^{\circ}$ to $173^{\circ}$ with a cosine diffuser. A pair of electrodes were positioned in the center of the cell to align the soot. In one experiment (Weiss et al. 1992) a square wave electric field of $\pm 2 \mathrm{kV} \mathrm{cm}^{-1}$ was applied to the plates 
for $2 \mathrm{~ms}$ at $12.5 \mathrm{~Hz}$. Both studies referenced above found an enhancement of $20 \%$ to 70 $\%$ when the soot is aligned with the electric field on.

It is of interest to compute the enhancement for the simulated soot. In both cases the investigators used a reciprocal type nephelometer where a cosine sensor/PM tube oriented normal to the laser beam collects scattered light from about $7^{\circ}$ to $173^{\circ}$. For this geometry, the integrated scattering cross section is related to scattering cross section given in Eq.(2) (Mulholland and Bryner 1994):

$$
\begin{aligned}
& C_{\text {sca, } a}^{\prime}=\int_{7^{\circ}}^{175^{\circ}}\left\langle\frac{d \sigma^{a g g}(q)}{d \boldsymbol{\Omega}}\right\rangle_{a} \sin \theta \mathrm{d} \theta, \\
& \text { where }\left\langle\frac{d \sigma^{a g g}(\mathbf{q})}{d \Omega}\right\rangle_{a}=N^{2} \frac{d \sigma^{m}}{d \Omega} S_{a}(q) \frac{1}{2}\left(1+\cos ^{2} \theta\right) .
\end{aligned}
$$

The prime is used to to distinguish this cross section from the total scattering cross section. The subscript $a$ refers to the partially aligned orientation and there is a parallel expression with subscript $r$ for the random orientation. The term $1 / 2\left(1+\cos ^{2} \theta\right)$ accounts for the Rayleigh scattering for the randomly polarized light source thought to be used in the experiments. This term was not included in our previous analysis, since the polarization direction was perpendicular to the scattering plane. The scattering cross section can be expressed as a product of the integral of a combined angular function $G_{a}(\theta)$ and an angle independent term denoted as $A$.

$$
C_{s c a, a}^{\prime}=\mathrm{A}_{7^{\circ}}^{173^{\circ}} G_{a}(\theta) d \theta \quad,
$$

where $G_{a}(\theta)=\left(1+\cos ^{2} \theta\right) \sin \theta S_{a}(\theta)$

and $A=\frac{1}{2} N^{2} \frac{d \sigma_{m}}{d \Omega}$.

Angular average scattering cross sections were computed for 300 sphere nanoagglomerates with $A_{31}$ equal 3.93, nearly the average value for 137 clusters, and $A_{31}$ equal 6.07, close to the sum of the average value plus the standard deviation. The monomer size parameter was the same as for the previous calculations $(\pi \mathrm{D} / \lambda=0.155)$. The peak in the combined angular function is larger for the partially aligned clusters compared to the randomly oriented clusters as indicated in Fig. 7. The ratio of $C_{s c a, a}^{\prime} / C_{s c a, r}^{\prime}$ is 1.76 for $A_{31}$ equal 3.93 and is 2.20 for $A_{31}$ equal 6.07. This increase in the ratio with increasing $A_{31}$ is the same trend as for the slope ratio $S R$. However, the integral in Eq.(33) is not related to $A_{31}$ in a straightforward manner. The observed ratio of cross sections (Colbeck et al., 1997 and Weiss et al.,1992) is in the range of 1.2 to 1.7.

The measured values are mostly less than either of our estimated values. One reason relates to the simulation parameters. Our simulation is based on nano clusters with 300 monomers. For the experimental conditions, there is likely a broad distribution of 
clusters extending to more than 1000 monomers. There is also the possibility that the principle axis of the cluster is not fully aligned in the direction of the electric field.

An existing light scattering instrument (Dick 2006) is capable of measuring $S(q)$ for a single particle over a time small compared to the rotation time of the particle for particles as small as $0.5 \mu \mathrm{m}$. If a single cluster were trapped at a fixed location, then $A_{31}$ could be determined from a series of measurements of $S(q)$ as the cluster rotates using an instrument such as Dick's. The ratio of the average slope (random orientation) to the minimum slope of $S(q)^{-1}$ vs. $q^{2}$ would be equal to $A_{31}$. The large $q$ behavior of $S(q)$ for a fixed orientation (see Figure 8) has multiple peaks and likely contains more information about the particle structure than $A_{31}$. These features arise from constructive and destructive interference from the scattered waves. For example, the first minimum shown in Figure 8 occurs for the major principal axis aligned with the y-axis, since the phase shift is approximately proportional to the y component of the radius of gyration (Eq.(11)). The orientation averaging (see Figure 6) removes these diffraction peaks.

The theory presented here is based on Rayleigh-Gans scattering. It ignores the coupling between the dipole fields from the monomers. Mulholland et al. (1994) found for clusters of size 17, 52, and 165 monomers, the difference between the prediction of the radius of gyration based on small angle Rayleigh-Debye-Gans theory and coupled electric and magnetic dipole theory range from $1 \%$ to $12 \%$ for the same size parameter $(2 \pi a / \lambda=0.15)$ as assumed in this study. For a fixed cluster size, it is likely that the ratio of the slopes will be less affected than the absolute value of the slope for different clusters. Still further analysis is needed to assess the effect of the coupled dipole analysis on the slope ratio.

\section{CONCLUSIONS}

A theory has been developed for computing the small angle light scattering by nanoclusters with fixed orientation and for nano-clusters aligned along their major principal axis. The ratio of the small angle slope for $1 / \mathrm{S}(\mathrm{q}) \mathrm{vs} . \mathrm{q}^{2}, S R$, is shown to be related to the principal components of the inertia matrix. The values of $S R$ were obtained from linear fits of Zimm plots for DLCA clusters in a process similar to what would be done experimentally. The resulting value of $S R$ was within $5 \%$ of the predicted asymptotic small angle value. It is also shown that $S R$ is well correlated with the ratio of the squares of the principle radii of gyration, $A_{31}$. For $S R$ over the range of 1.5 to 9 , the standard deviation from the regression line is \pm 0.06 . This demonstrates the unique possibilities of small $\mathrm{q}$ light scattering with partially aligned and random particles to determine the particle shape.

For large q for nano-clusters of with 30, 100, 300, and 1000 monomers, it is shown from the simulations that $\mathrm{S}(\mathrm{q})$ for the partially aligned clusters is not proportional to $q^{-D_{f}}$ as it is for the randomly oriented clusters. Nano-cluster with a fixed orientation are shown to produce diffraction peaks. Calculation of the integrated light scattering cross sections for 300 sphere nano-clusters indicate a significant increase of $70 \%$ and $120 \%$ for partially 
aligned versus randomly oriented clusters for two representative aspect ratios. The smaller value overlaps with measured values of the scattering enhancement for oriented soot agglomerates in an electric field (Colbeck et al. 1997 and Weiss et al. 1992).

\section{REFERENCES}

Allen, M., and Tildesly, D. (1987). Computer Simulation of Liquids. Clarendon Press, Oxford.

Chen, M. T., Xie, G.W., Yang M., and Shaw D. T. (1991). Experimental Characterization of ChainAggregate Aerosol by Electrooptic Scattering. Aerosol Sci. \& Technol., 14: 74-81

Colbeck, I., Atkinson, B., and Johart Y. (1997). The Morphology and Optical Properties of Soot Produced by Different Fuels. J. Aerosol Sci., Vol. 28, 715-723.

Dick, W.D. (2006). Internal report by MSP Corp on multi-angle light scattering photometer, Shoreview, MN.

Family, F., and Landau, D. P. (eds.) (1984). Kinetics of Aggregation, and Gelation. North Holl, and Elsevier, Amsterdam.

Fry, D., Mohammad, A., Chakrabarti, A., and Sorensen, C. M. (2004). Cluster Shape Anisotropy in Irreversibly Aggregating Particulate Systems. Langmuir, 20:7871.

Guinier, A. (1939). La Diffraction Des Rayons X Aux Trespetits Angles: Application a L'etude de Phenomes Ultramicroscopiques, Ann. Phys., 12:161-237.

Jullien, R., and Botet, R. (1987). Aggregation, and Fractal Aggregates, World Scientific, Hackensack, New Jersey.

Heinson, W. R., Sorensen, C.M., and A. Chakrabarti, A. (2010). Does Shape Anisotropy Control the Fractal Dimension in Diffusion-Limited Cluster-Cluster Aggregation? Aerosol Sci. \& Technol., 44: iiv.

Kerker, M. (1969). The Scattering of Light and Other Electromagnetic Radiation, Academic, New York.

Martin, J. E., and Hurd, A. J. (1987). Scattering from Fractals, J. Appl. Cryst., 20:61-78

Meakin, P. (1999). A Historical Introduction to Computer Models for Fractal Aggregates. J. Sol. Gel. Sci. Technol., 15:97.

Mountain, R.D. and Mulholland, G.W. (1988). Light Scattering from Simulated Smoke Agglomerates. Langmuir, 4, 1321.

Mulholland, G. W. and Bryner, N. P. (1994a). Radiometric Model of the Transmission Cell-Reciprocal Nephelometer. Atmospheric Environment, 28, 873-887.

Mulholland, G. W., Bohren, C. F., Fuller, K. A. (1994b). Light Scattering by Agglomerates: Coupled Electric and Magnetic Dipole Method. Langmuir, 10, 2533-2546.

Sorensen, C. M. (2001). Light Scattering by Fractal Aggregates: A Review. Aerosol Sci. Technol., 35:648 687.

Tanford, C. (1961). Physical Chemistry of Macromolecules, Wiley, New York.

Weiss, R. E., Kapustin, V. N. and Hobbs, P. V. (1992) Chain-Aggregate Aerosols in Smoke from the Kuwait Oil Fires. J. Geophys. Res., 97: 14,527-14,531.

Zimm, B. H. (1948). Apparatus and Methods for Measurement and Interpretation of the Angular Variation of Light-Scattering, Preliminary Results on Polystyrene Solutions, J. Chem. Phys., 16:1099-1116. 


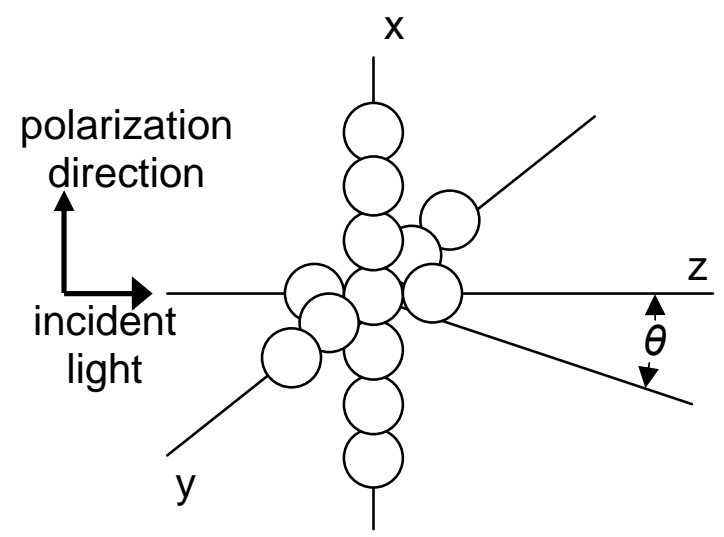

FIG. 1. Schematic of light scattering geometry with the $z-y$ plane the scattering plane. The orientation of a simple cluster with the major principal axis along the $x$-direction and the minor principal axis in the $z$-direction is shown.
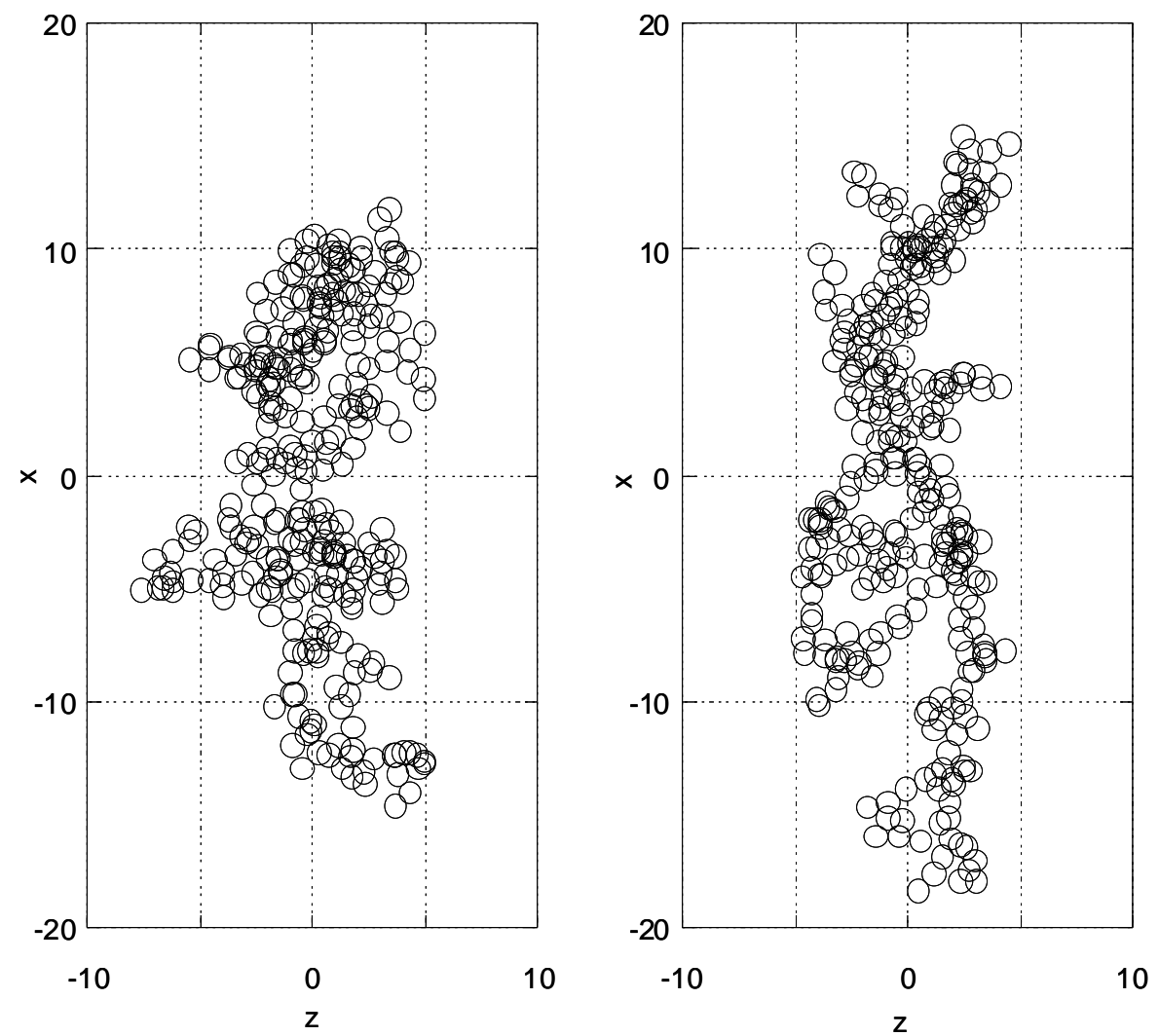

FIG. 2. x-z projection of 300 sphere clusters with $A_{31}=2.13$ (left) and 6.07 (right). 


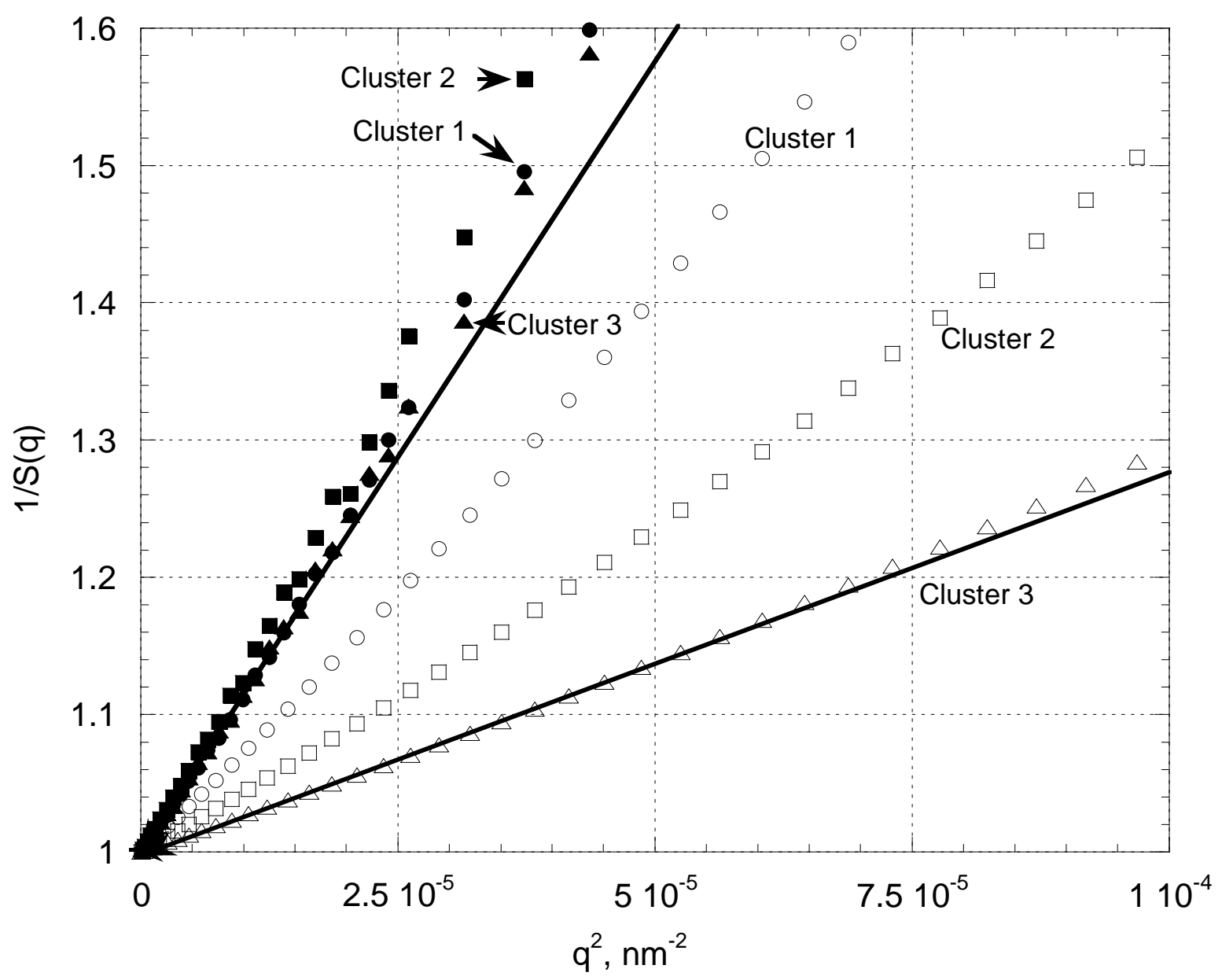

FIG. 3. Effect of shape on $1 / S(q)$ for 300 sphere nano-clusters, $A_{31}=2.18$ cluster 1 , $A_{31}=4.35$ cluster $2, A_{31}=6.54$ cluster 3 . Open symbols refer to partially aligned particles and the solid to the randomly oriented. The solid lines are visual fits for values of $1 / S(q)$ less than 1.2. 
Table 1. Comparison of $S R$ based on cluster coordinates and SR from small $q$ behavior of $1 / S(q)$ for cluster with 30 to 1000 monomers generated by DLCA

\begin{tabular}{|c|c|c|c|c|c|}
\hline$N$ & $R g, \mathrm{~nm}$ & $A_{31}$ & $S R(\mathrm{Eq} 23)$ & $S R($ fit $)$ & $\% \operatorname{diff}^{a}$ \\
\hline 31 & 50.1 & 2.18 & 1.63 & 1.59 & -2.8 \\
\hline 30 & 63.2 & 4.35 & 3.01 & 2.96 & -1.6 \\
\hline 30 & 71.9 & 6.54 & 4.60 & 4.47 & -2.9 \\
\hline 307 & 180 & 2.13 & 1.54 & 1.56 & 0.9 \\
\hline 301 & 192 & 3.93 & 2.89 & 2.87 & -0.5 \\
\hline 304 & 183 & 6.07 & 4.30 & 4.17 & -3.1 \\
\hline 100 & 92 & 2.06 & 1.67 & 1.57 & -6.0 \\
\hline 100 & 128 & 3.86 & 2.67 & 2.63 & -1.5 \\
\hline 1007 & 337 & 2.08 & 1.53 & 1.51 & -1.6 \\
\hline 989 & 369 & 4.10 & 2.99 & 3.04 & 1.8 \\
\hline
\end{tabular}




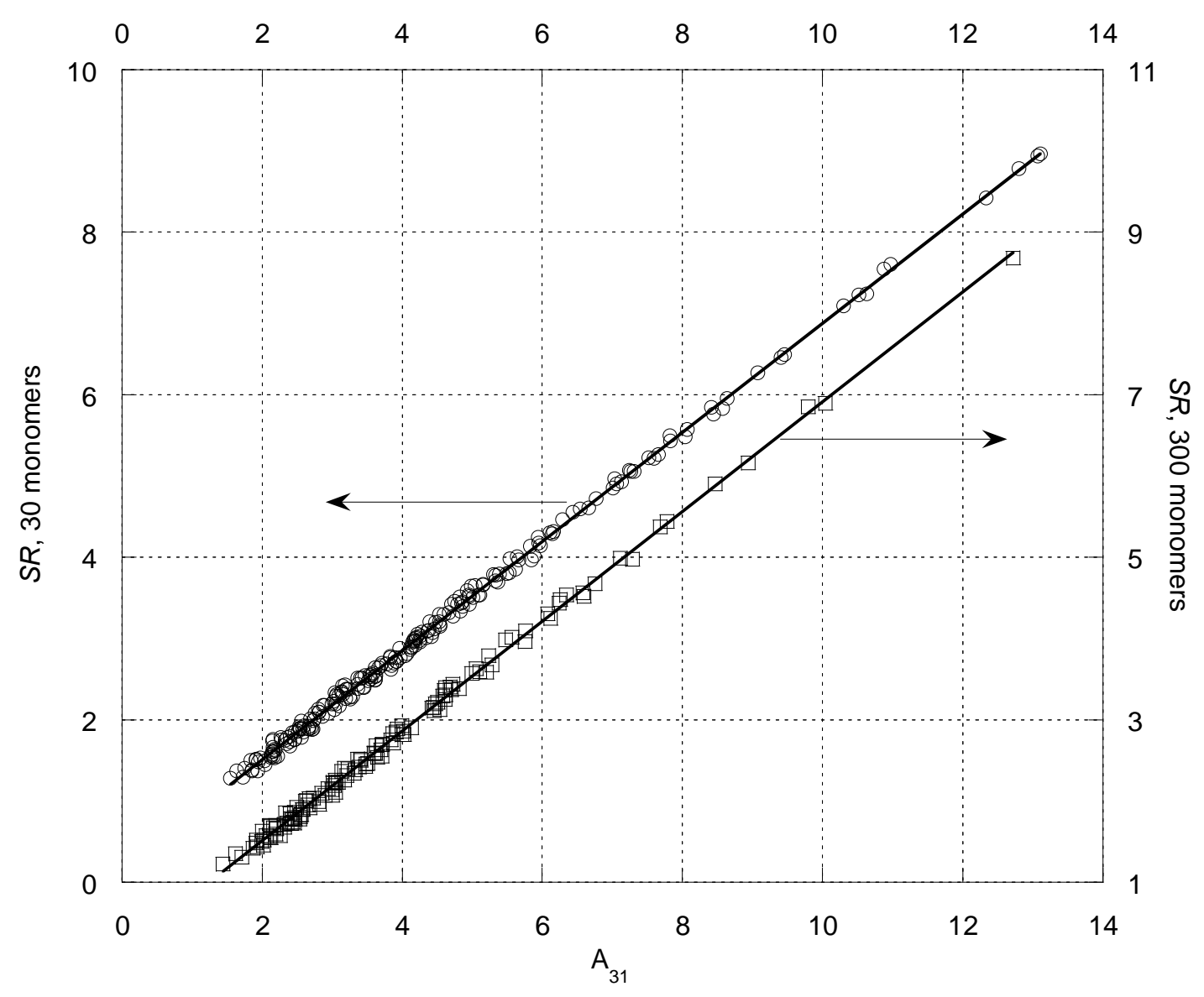

FIG. 4. Linear regression of $S R$ versus anisotropy for 227 clusters with 30 monomers and 137 clusters with 300 monomer produced by DLCA. The axis is offset on the right hand side to allow viewing of both correlation lines. The mean and standard deviation for $S R$ and $A_{31}$ are $3.11 \pm 1.52$ and $4.38 \pm 2.27$ for 30 monomer clusters and $2.82 \pm 1.53$ and $3.94 \pm 2.27$ for the 300 monomer clusters. 


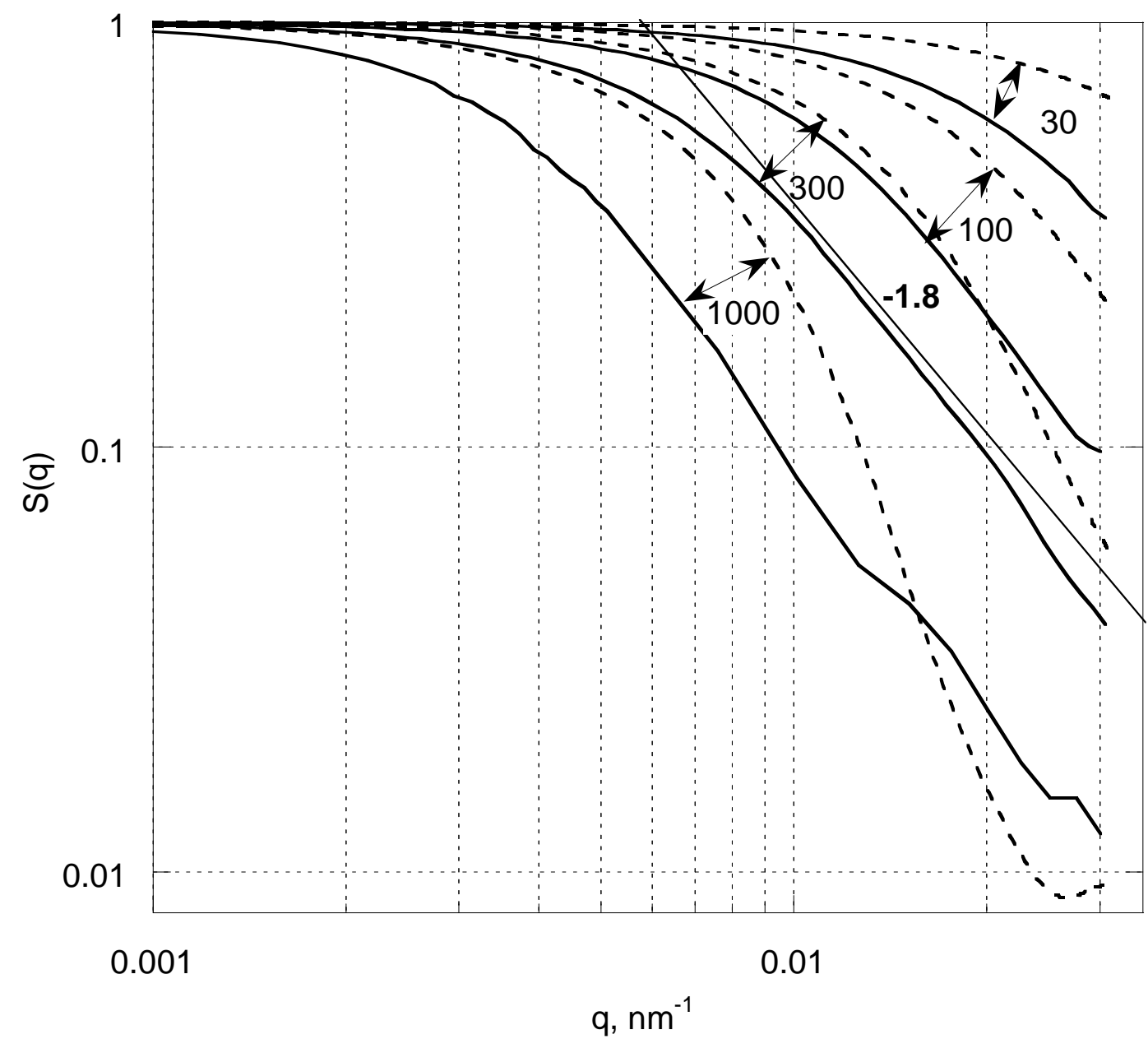

FIG. 5. The effect of the number of monomers in the cluster on the structure factor for a wide range in $q$. The value of $A_{31}$ in all four cases is near 4.0. The solid lines correspond to a random orientation and the dashed lines to a partially aligned orientation. A faint solid line is drawn with a slope corresponding to a fractal dimension of 1.8. 

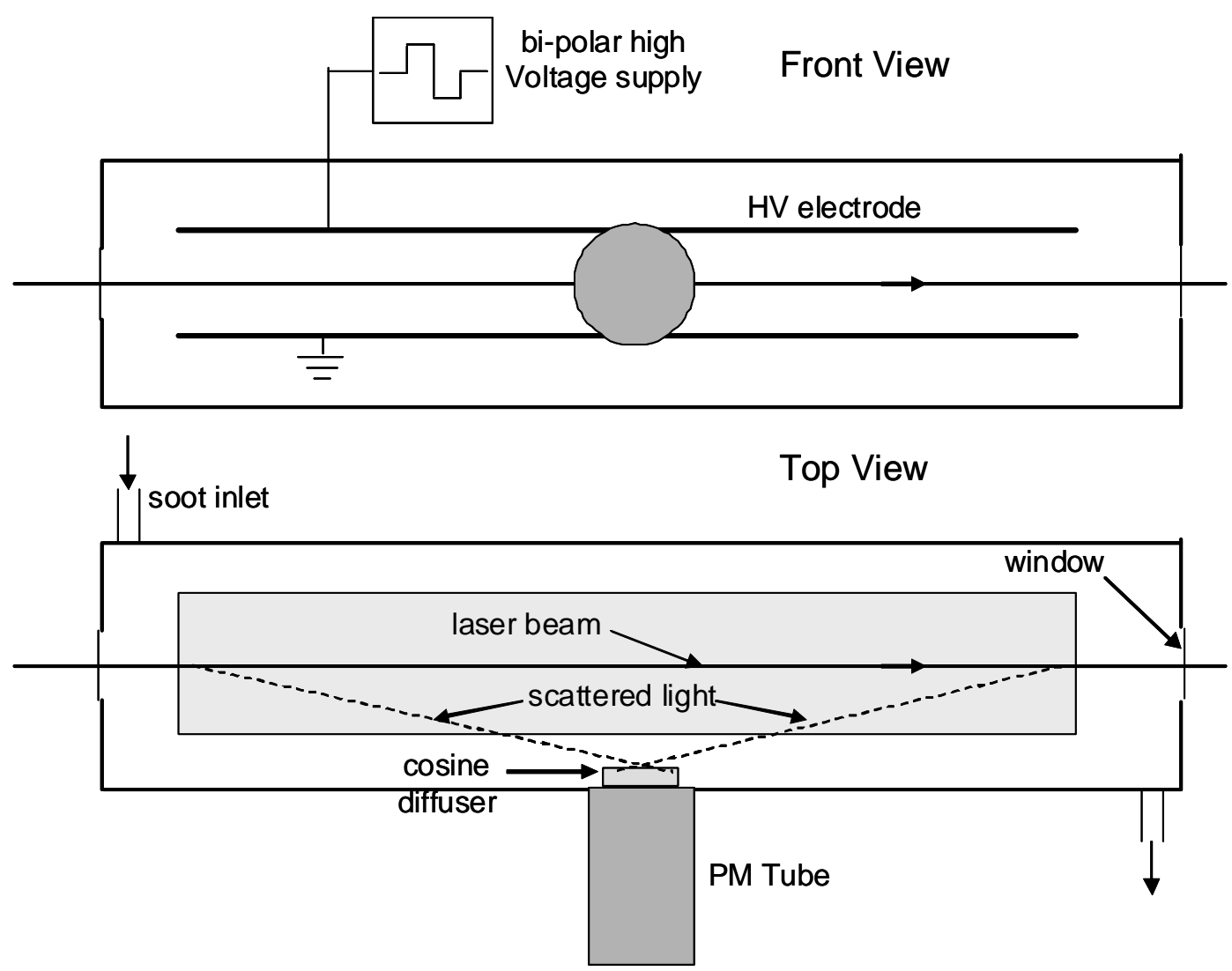

FIG. 6. Schematic of a reciprocal nephelometer with inserted electrodes for orienting the agglomerates. The scattered light is collected by the cosine diffuser over an angle range of about $7^{\circ}$ to $173^{\circ}$. The shaded section in the top view represents the electrode. 


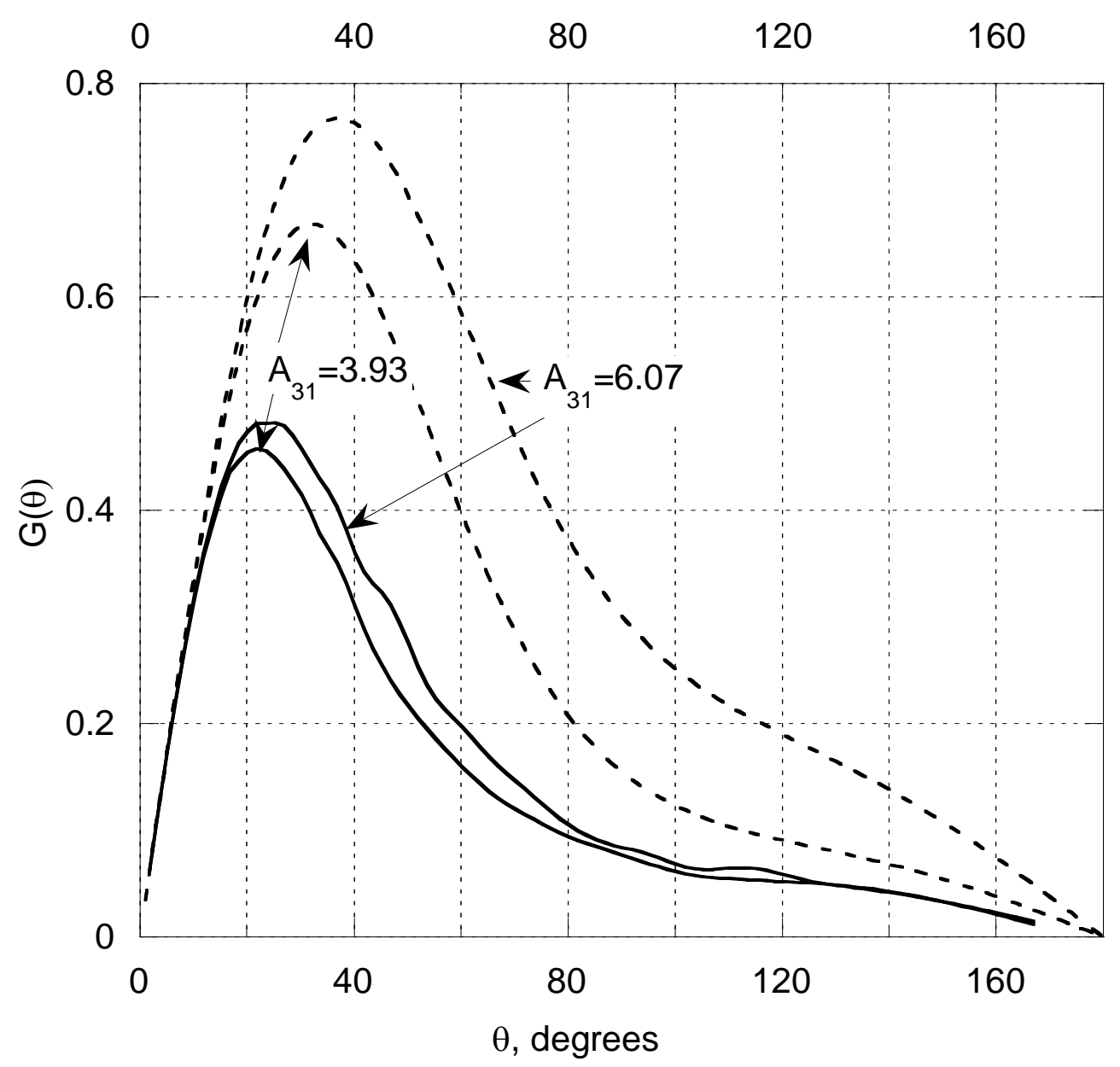

FIG. 7. The angular dependence of the combined angular function for the partially aligned 300 monomer nano-cluster shown by dashed curves and the random orientated nano-cluster shown by the solid line. For each orientation results are given for two values of the anisotropy $A_{31}$. 


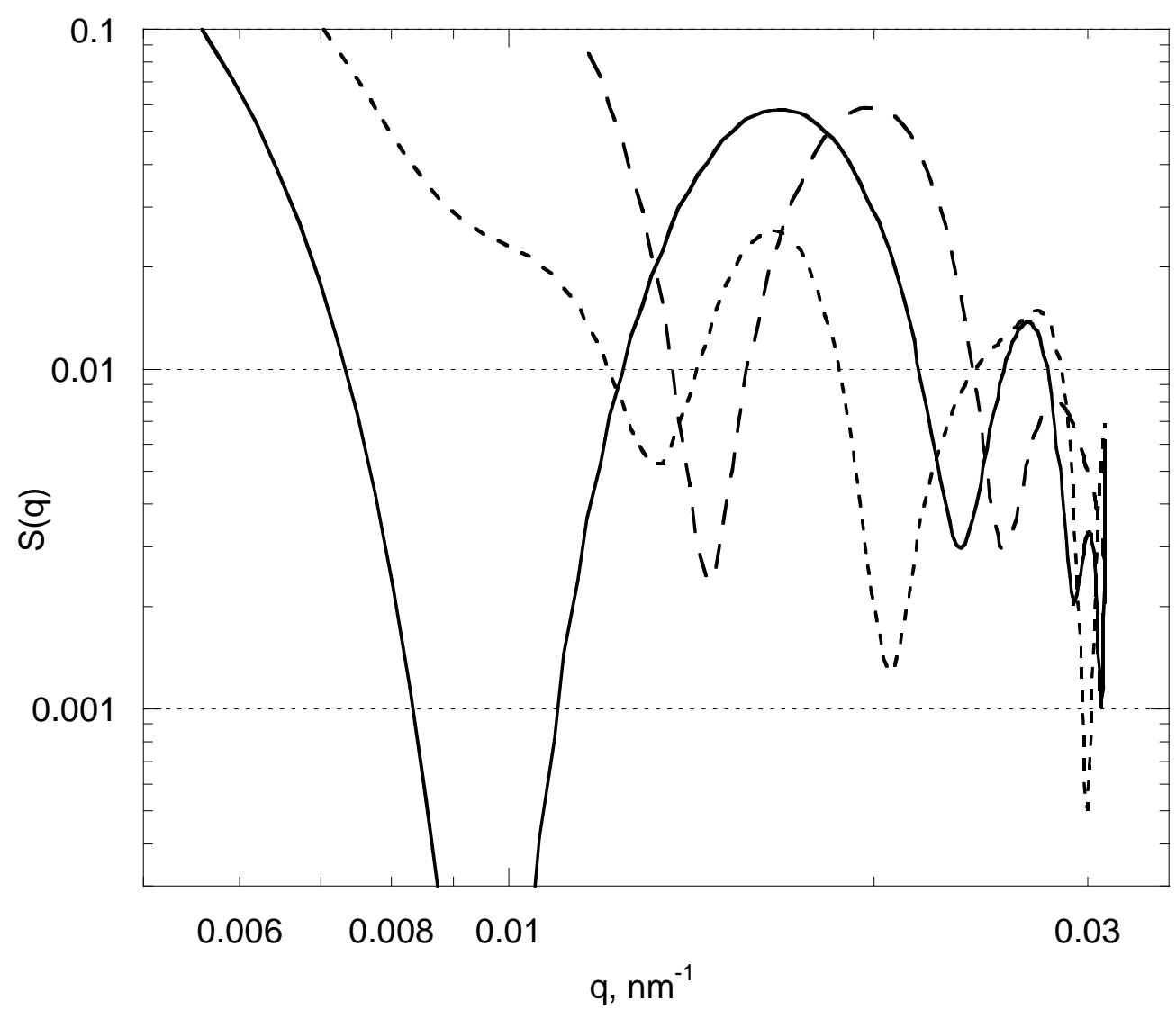

FIG. 8. $S(\mathrm{q})$ is plotted for a nano-cluster with 1007 monomers. Each curve corresponds to a different orientation of the cluster. The solid curve corresponds to the largest principle axes in the y-direction, the short dashed curve for largest principle axis in the zdirection, and the long dashed curve for largest principle axis in the x-direction. In all three cases the light is propagating in the $\mathrm{z}$-direction and polarized in the $\mathrm{x}$-direction with the $y-z$ plane the scatter plane. 